\title{
Epitope spreading induced by immunization with synthetic SSB peptides
}

\author{
MIN DING $^{1,2}$ and JIANZHONG ZHANG ${ }^{1}$ \\ ${ }^{1}$ Department of Dermatology, Peking University People's Hospital, Beijing 100044; \\ ${ }^{2}$ Department of Dermatology, Xuzhou Central Hospital, Xuzhou, Jiangsu 221009, P.R. China
}

Received November 10, 2015; Accepted April 18, 2016

DOI: $10.3892 /$ etm.2016.3267

\begin{abstract}
Sjogren's syndrome type B (SSB)/La antibody is an autoantibody generally observed in connective tissue diseases whereas double-stranded deoxyribonucleic acid (dsDNA) antibodies are the most characteristic autoantibodies found in systemic lupus erythematosus (SLE) patients. The relationship of these autoantibodies remains unclear. The aim of the study was to determine the profile of antibody production in rabbits immunized with synthetic SSB peptides alone or with dsDNA. For this purpose, 214-225aa peptide of SSB antigen was synthesized based on the organic chemistry solid-phase peptide synthesis. Rabbits were immunized with the following antigens: i) synthetic SSB peptides linked with keyhole limpet hemocyanin (KLH); ii) dsDNA; iii) SSB plus dsDNA; iv) KLH; and v) phosphate-buffered saline. SSB peptide antibody was measured using the enzyme-linked immunosorbent assay while extractable nuclear antigens (ENA) antibody and dsDNA antibody were measured by immunoblotting and immunofluorescence, respectively. The results showed that a specific anti-SSB peptide antibody was produced following immunization with SSB epitope alone or with dsDNA. The SSB peptide antibody titer in the coimmunization group was higher than that of the SSB alone group. In addition, antibodies against ribonucleoprotein (RNP), Smith and/or dsDNA were
\end{abstract}

Correspondence to: Dr Jianzhong Zhang, Department of Dermatology, Peking University People's Hospital, 11 Xizhimen South Street, Beijing 100044, P.R. China

E-mail: dingmin_9@163.com

Abbreviations: CFA, Freund's complete adjuvant; DMF, dimethylformamide; dsDNA, double-stranded deoxyribonucleic acid; ELISA, enzyme-linked immunosorbent assay; ENA, extractable nuclear antigen; FITC, fluorescein isothiocyanate; IFA, Freund's incomplete adjuvant; KLH, keyhole limpet hemocyanin; PBS, phosphate-buffered saline; RNP, ribonucleoprotein; SLE, systemic lupus erythematosus; SSA, Sjogren's syndrome type A antigen; SSB, Sjogren's syndrome type B antigen

Key words: Sjogren's syndrome type B antigen peptides, double-stranded deoxyribonucleic acid, co-immunization, epitope spreading detected in rabbits of the coimmunization group. The presence of anti-dsDNA antibodies in the rabbits immunized with SSB peptide suggested the induction of epitope spreading. In conclusions, SSB antibodies were produced in rabbits immunized with SSB peptide or SSB+dsDNA, whereas SSB antibody titers were higher in the coimmunization group. Furthermore, coimmunization was associated with epitope spreading.

\section{Introduction}

Sjogren's syndrome type B antigen (SSB)/La antibody is one of the autoantibodies generally observed in the connective tissue diseases such as Sjögren's syndrome, neonatal lupus erythematosus and sub-acute cutaneous lupus erythematosus. Patients with anti-SSB antibodies are more likely to experience less severe conditions and improved prognosis $(1,2)$. On the other hand, double-stranded deoxyribonucleic acid (dsDNA) antibodies were identified as the most characteristic autoantibodies in patients with systemic lupus erythematosus (SLE) and their vital roles in the pathogenesis of lupus and other autoimmune diseases have been described (3-5). In general, multiple autoantibodies may be detected simultaneously in patients with lupus erythematosus. Smith $(\mathrm{Sm})$ antibodies are often associated with u1-ribonucleoprotein (RNP) antibodies whereas SSA antibodies are usually found with SSB antibodies. This type of antibody co-prevalence was related to the phenomenon of epitope spreading, during which, epitopes that are non-crossreactive and distinct from an inducing epitope become the main target of the ongoing immune response e.g., as a result of acute/persistent infection or secondary to chronic tissue destruction during progressive autoimmune disease (5). The relationship among these autoantibodies remains unclear. In the present study, we determined the antibody profile in rabbits that were immunized with synthetic SSB peptides alone or in combination with dsDNA. The results showed that beside the production of target antibodies, coimmunization was associated with epitope spreading.

\section{Materials and methods}

Reagents. The main reagents used in this study included: fluorenylmethyloxycarbonyl (Fmoc) amino acids (Amersham Pharmacia Biotech, Piscataway, NJ, USA), hemocyanin (Sigma-Aldrich, Hong Kong, China), calf thymus DNA 
(DingGuo ChangSheng Biotechnology Co., Ltd., Beijing, China), and dsDNA immunofluorescence assay kit and extractable nuclear antigens (ENA) polypeptide spectrum kit (Euroimmun AG, Lübeck, Germany).

Prediction and design of amino acid sequences containing polypeptide antigens. The primary sequence of SSB/La protein was obtained based on web-based resources, and prediction and analysis were conducted by using specific structural analysis software. An SSB/La polypeptide was designed and synthesized after comprehensive consideration of its secondary structure, hydrophilicity, and surface accessibility in combination with immunogenic epitopes. The sequence of this polypeptide was: SSB/La 214-225aa KQKLEEDAEMKS-Y. The C-terminal tyrosine (Y) was coupled with keyhole limpet hemocyanin (KLH) vector to yield the KQKLEEDAEMKS-Y-KLH conjugate.

Synthesis and purification of epitope-containing polypeptide. The target polypeptide with antigenic epitopes was synthesized from C- to N-terminus by using the solid-phase synthesis approach (with Fmoc-protected amino acids as the starting materials on solid supporter-resin) using an automated peptide synthesizer (ABI Mode 433A; Applied Biosystems, Foster City, CA USA). The yielded peptide-resin was hydrolyzed to obtain the peptide crude product. The crude product was then purified by C18-reverse phase high-performance liquid chromatography (600E; Waters Corp., Milford, MS, USA) and the purified product $(>95 \%)$ was freeze-dried (Virtis Company, Inc., Cardiner, NY, USA).

Rabbit immunizations. For this purpose, 10 healthy New Zealand white rabbits (average body weight: $\sim 2 \mathrm{~kg}$ ) were procured from the animal hold unit, at the People's Hospital affiliated to Peking University (Beijing, China) and randomized into 5 groups, containing 2 rabbits each: i) SSB immunization group, animals were labeled as SSB1 and SSB2; ii) dsDNA immunization group, animals were labeled as DNA1 and DNA2; iii) SSB+dsDNA immunization group, animals were labeled as co-immunization 1 and co-immunization 2; iv) KLH immunization group, animals were labeled as KLH1 and KLH2; and v) phosphate-buffered saline (PBS) group, animals were labeled as control no. 1 and control no. 2 .

Rabbits were immunized with SSB polypeptide at a concentration of $1 \mathrm{mg} / \mathrm{ml}$. During the primary immunization, $1 \mathrm{ml}$ of antigenic polypeptide was emulsified with $1 \mathrm{ml}$ of complete Freund's adjuvant (CFA). For emulsification, one part of adjuvant and one part of antigen were aspirated into a syringe and emulsified by repeated plunger movements. A successful emulsification was yielded when no spreading of emulsified droplets was observed in water. The mixture was administered subcutaneously through multiple injections (40-50 injection sites) in the back. Booster immunization comprised the antigenic polypeptide and incomplete Freund's adjuvant (IFA). Rabbits were immunized for 4 times with a 20-day interval. After 2 weeks of the 4th immunization, rabbits were sacrificed by exsanguination.

Enzyme-linked immunosorbent assay (ELISA). Blood samples were collected at 10 days following the $2 \mathrm{nd}$, 3rd and 4th immunizations and serum samples were separated. Serum antibody titers were determined by indirect ELISA. Antigenic SSB peptide fragment (free of hemocyanin; $0.2 \mu \mathrm{g} / 0.1 \mathrm{ml} / \mathrm{well}$ ), DNA ( $2 \mu \mathrm{g} / 0.1 \mathrm{ml} /$ well) and hemocyanin in carbonate buffer (pH 9.6) were added to 96-well microtiter plates and incubated at $4^{\circ} \mathrm{C}$ overnight, followed by washing with PBS-T ( $0.01 \mathrm{M}$ PBS pH 7.4 with $0.05 \%$ Tween-20) 4 times and dried. Plates were blocked by incubation at $37^{\circ} \mathrm{C}$ for $1 \mathrm{~h}$ with $1 \%$ bovine serum albumin (BSA) in PBS-T buffer (pH 9.6; $0.1 \mathrm{ml} /$ well), followed by washing 4-5 times and dried. Rabbit serum was diluted in $1 \%$ BSA PBS-T buffer to give the following dilutions: 1:100; $1: 200 ; 1: 400 ; 1: 800 ; 1: 1,600 ; 1: 3,200$; and 1:6,400. Using normal rabbit serum (1:1,000 dilution; $0.1 \mathrm{ml} /$ well) as negative control, the plates were incubated at $37^{\circ} \mathrm{C}$ for $1 \mathrm{~h}$, followed by repeated washes as before. Then, secondary antibody i.e., horseradish peroxidase-labeled goat anti-rabbit immunoglobulin $\mathrm{G}(\mathrm{IgG})$ was added $(0.1 \mathrm{ml} /$ well $)$ and incubated at $37^{\circ} \mathrm{C}$ for $40 \mathrm{~min}$, followed by repeated washes. Chromogenic reaction was developed by incubating for $10 \mathrm{~min}$ in the dark at $37^{\circ} \mathrm{C}$ with solution A (10 mg temporomandibular disorder $+1 \mathrm{ml}$ dimethylformamide), diluted 1:100 with citrate buffer ( $\mathrm{pH}$ 5.0) and solution $\mathrm{B}\left(12 \mathrm{ml}\right.$ distilled water $\left.+5 \mathrm{ml} \mathrm{H}_{2} \mathrm{O}_{2}\right)$. The reaction was terminated with $2 \mathrm{~N} \mathrm{H}_{2} \mathrm{SO}_{4}$ by adding 2 drops/well and optical density (OD) was read at $450 \mathrm{~nm}$ ultraviolet wavelength. OD values $>1.5$-fold the negative control OD were considered as positive. For positive results, the highest dilution was considered to be the antibody titer.

ENA and dsDNA antibody assays. ENA antibody assay was performed using ENA polypeptide spectrum kit, based on western blotting, according to the manufacturer's instructions. Similarly, dsDNA antibody assay was carried out using dsDNA assay kit, based on fundamental procedure of indirect immunofluorescence assay, according to the manufacturer's instructions.

\section{Results}

Anti-SSB polypeptide antibody titers. Serum samples were collected at the 2nd, 3rd and 4th immunization and anti-SSB polypeptide antibodies were measured by ELISA. Although no anti-SSB polypeptide antibodies were detected in PBS, hemocyanin, and the dsDNA immunization groups, anti-SSB polypeptide antibody titers at $2 \mathrm{nd}, 3 \mathrm{rd}$ and 4 th immunizations in rabbit SSB1 were 1:800, 1:1,600 and 1:3,200, respectively. The corresponding antibody titers in rabbit SSB2 were 1:100, 1:200 and 1:800, respectively. In the SSB+dsDNA co-immunization group, respective anti-SSB titers in the co-immunization group rabbit 1 were 1:3,200, 1:6,400 and $1: 6,400$, and in co-immunization group rabbit 2 were 1:1,600, $1: 6,400$ and 1:6,400. Thus, anti-SSB polypeptide antibody titers in the co-immunization groups were significantly higher than those of the SSB alone group at each time point (Fig. 1).

ENA and dsDNA antibody profiles. The induction of anti-SSB polypeptide antibody was concomitant with the positive detection of anti-Sm antibody in the co-immunization group rabbit 1 and with anti-RNP and anti-Sm antibodies in co-immunization group rabbit 2. A positive detection of anti-dsDNA antibody in rabbit SSB1 (1:160 titer; Fig. 2) was consistent with its detection 


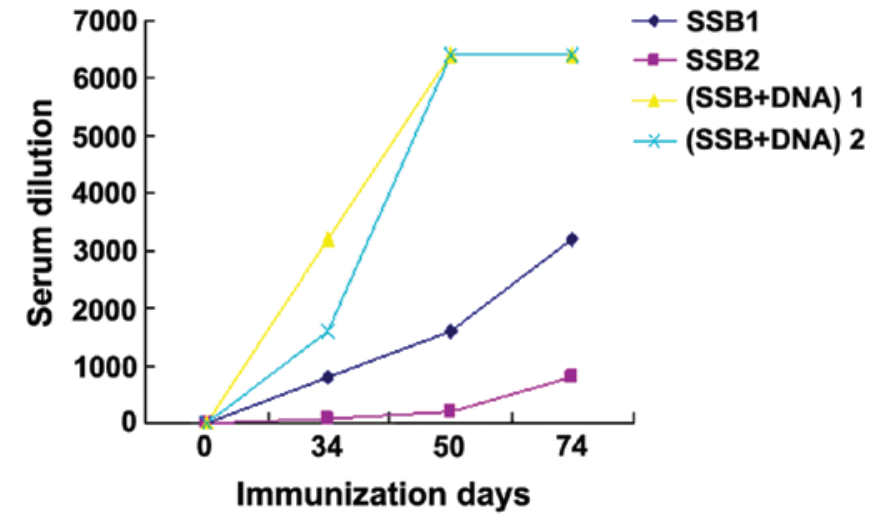

Figure 1. Anti-Sjogren's syndrome type B (SSB) polypeptide antibody titers following immunization with SSB polypeptide alone or in combination with double-stranded deoxyribonucleic acid (dsDNA). As determined by ELISA anti-SSB polypeptide antibody titers were significantly higher in rabbits immunized with SSB+dsDNA as compared with those immunized with SSB polypeptide alone.

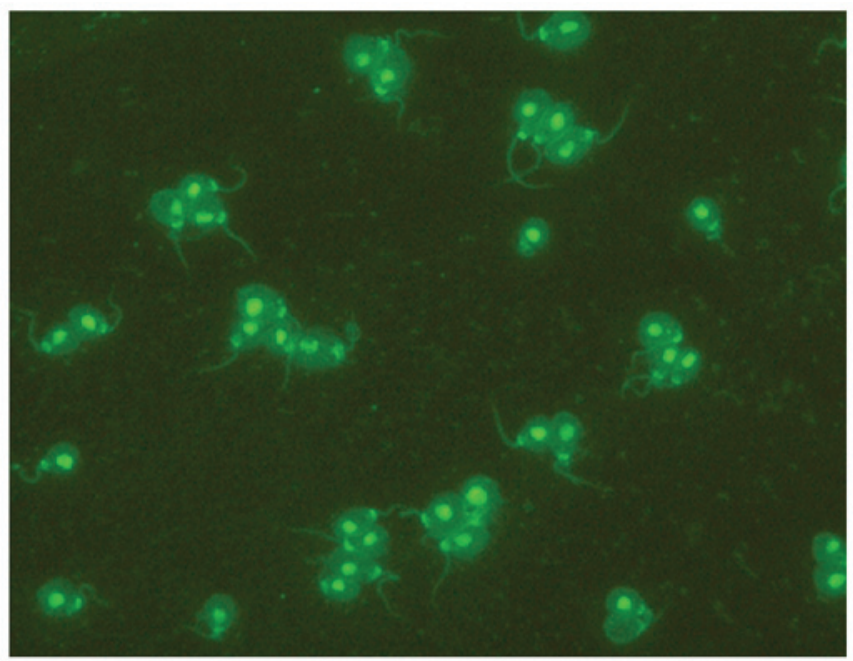

Figure 2. Indirect immunofluorescence detection test for anti-double-stranded deoxyribonucleic acid antibodies. A bright green fluorescence of crithidia luciliae was observed in the Sjogren's syndrome type B immunization group (using a serum dilution of 1:160).

in the co-immunization group rabbit 1 . No anti-dsDNA antibody was detected in rabbits from the other groups.

\section{Discussion}

Autoantibodies of different types and clinical relevance are commonly found in the sera of patients with SLE and Sjögren's syndrome (5). Certain autoantibodies even play a role in the pathogenesis of diseases (5). It is, however, unclear whether any antibody interactions occur during the course of their production in the host. In the present study, SSB and dsDNA antigens were used, alone or in combination, to immunize rabbits and determine their antibody profiles. First, the human SSB/La antigen (214-225aa) corresponding to the immunodominant epitope region (179-242aa) was selectively designed and synthesized by following standard peptide synthesis method (6-10). The result showed that anti-SSB polypeptide antibodies were produced in the SSB and SSB+dsDNA coimmunization groups, indicating the presence of epitopes in the synthesized polypeptide fragment. Anti-SSB antibody peak titers were observed at the 3rd immunization in the SSB+dsDNA group. In the SSB group, relatively lower antibody titers were observed. Mammalian chromatin, nucleosomes, DNA, and histones rarely induce autoimmune responses (11-13), and $\mathrm{CD}^{+}$and $\mathrm{CD}^{+}{ }^{+} \mathrm{T}$-cell populations with suppressive activities against anti-DNA antibody production were observed in normal mice, but not in mice with lupus (14). Of note, we also did not detect anti-dsDNA antibodies following immunization with dsDNA alone, suggesting that the dsDNA was incapable of inducing antibody production. Notably, although the DNA had no immunogenicity, anti-SSB antibody titers were higher in the SSB+dsDNA coimmunization group as compared with the SSB group. It may be possible that the coimmunization with DNA antigen had enhanced the immunogenicity of SSB antigen or induced the antigenic stimulation for higher antibody production.

In the current study, the rabbits also produced RNP and Sm antibodies in addition to anti-SSB polypeptide antibodies following co-immunization. Furthermore, anti-dsDNA antibodies were detected in SSB1 and coimmunization 1 rabbits, suggesting the induction of intra- and inter-molecular epitope spreading following SSB plus dsDNA coimmunization. Since the mammalian DNA is devoid of auto-immunogenicity, the induction of anti-dsDNA antibody may, therefore, be a result of SSB polypeptide-induced epitope spreading. Similar findings of SSB antigen-induced epitope spreading have been reported previously (15). Epitope spreading is defined as the development of immune response to a specific epitope as well as other epitopes in the early stages of T- or B-cell-mediated responses, spanning from monoclonal or oligoclonal to polyclonal $\mathrm{T}$ or $\mathrm{B}$ cell activation. From the perspective of anti-infection, epitope spreading is considered an efficient mechanism to generate effective immune response against alien epitopes of invading pathogens or tumor cells.

Notably, an autoimmune response may also be triggered by exogenous antigen(s) that progresses to a truly autoimmune reaction, resulting in the induction of self-epitope specific antibodies and lymphocytes. Epitope spreading may occur from dominant epitopes to subdominant or recessive epitopes, from intra- to intermolecular epitopes, or from exoepitopes (e.g., viruses) to endoepitopes. Epitope spreading may occur at the following positions: i) inside of an antigenic molecule i.e., intramolecular; ii) different antigen or antigenic determinants inside of a macromolecule, i.e., related intermolecular; and iii) epitopes with no direct interconnections and special anatomical distribution under physical or physiological conditions i.e., intermolecular. These types of epitope spreading have been previously reported (16-19). For instance, the presence of cross-reactivity between Epstein-Barr virus (EBV) nuclear antigen-1 during EBV infection and Ro, Sm B/B' and Sm D1 antigens was reported in lupus patients (16). A cross-reactivity between Ro60 and SmD autoantigens (17) as well as antigenic cross-reactivity among full-length SSB, other epitopes, and myelin (18) were also reported. SSB antigen-immunized animals developed intra- or intermolecular epitope spreading (17-19). High titers of anti-dsDNA IgG antibodies were observed, in addition to anti-histone and anti-cardiolipin antibodies, in BALB/c mice immunized with 
DWEYSVWLSN and adjuvant (20). Scofield et al (21) reported the development of Ro intramolecular immune spreading and intermolecular epitope spreading for SSB, ds DNA, nRNP and $\mathrm{Sm}$ in animals immunized with HNE-Ro antigen. Epitope spreading can be related to multiple factors, such as the physical characteristics of antigens, the presence of modified antibody, effect of genetic background, and the established level of immune tolerance. It was suggested that the intermolecular immune spreading was antigen-dependent while the induction of an autoimmune response was antigen-driven (22). Epitope spreading is widely presented in autoimmune diseases, which are closely associated with their pathogenesis.

In conclusion, the findings show that after rabbit coimmunization with SSB polypeptide and dsDNA, besides the target antibody production, unexpected antibodies (anti-ENA and anti-dsDNA) were also induced, suggesting the intra- and intermolecular epitope spreading. However, in-depth studies are required to elucidate the mechanisms and pathogenesis of autoimmune diseases.

\section{Acknowledgements}

The present study was supported by grant from the National Natural Science Foundation (no. 30271194). We would like to thank Professor Qianjin Lu from Central South University for critical comments and technical review of the manuscript.

\section{References}

1. Wasicek CA and Reichlin M: Clinical and serological differences between systemic lupus erythematosus patients with antibodies to Ro versus patients with antibodies to Ro and La. J Clin Invest 69: 835-843, 1982.

2. Zhang JZ, Jin J, Guo LS and Ma SQ: Different prognoses of two types of sub-acute cutaneous lupus erythematosus: A follow-up of 40 cases. Chin J Dermatol 34: 9-11, 2001.

3. Balow JE and Austin HA III: Renal disease in systemic lupus erythematosus. Rheum Dis Clin North Am 14: 117-133, 1988.

4. Arbuckle MR, James JA, Kohlhase KF, Rubertone MV, Dennis GJ and Harley JB: Development of anti-dsDNA autoantibodies prior to clinical diagnosis of systemic lupus erythematosus. Scand J Immunol 54: 211-219, 2001.

5. Schiffer LE, Hussain N, Wang X, Huang W, Sinha J, Ramanujam $M$ and Davidson A: Lowering anti-dsDNA antibodies - what's new? Lupus 11: 885-894, 2002.

6. Chambers JC, Kenan D, Martin BJ and Keene JD: Genomic structure and amino acid sequence domains of the human La autoantigen. J Biol Chem 263: 18043-18051, 1988.

7. Kenan DJ, Query CC and Keene JD: RNA recognition: Towards identifying determinants of specificity. Trends Biochem Sci 16: 214-220, 1991.
8. Gordon TP, Greer M, Reynolds P, Guidolin A and McNeilage LJ: Estimation of amounts of anti-La(SS-B) antibody directed against immunodominant epitopes of the $\mathrm{La}(\mathrm{SS}-\mathrm{B})$ autoantigen. Clin Exp Immunol 85: 402-406, 1991.

9. McNeilage LJ, Macmillan EM and Whittingham SF: Mapping of epitopes on the La(SS-B) autoantigen of primary Sjögren's syndrome: Identification of a cross-reactive epitope. J Immunol 145: 3829-3835, 1990.

10. Kohsaka H, Yamamoto K, Fujii H, Miyasaka N, Miura H, Tanaka Y, Nishioka K and Miyamoto T: Molecular cloning of cDNAs expressing SS-B/La protein. J Autoimmun 2: 353-357, 1989.

11. Madaio MP, Hodder S, Schwartz RS and Stollar BD: Responsiveness of autoimmune and normal mice to nucleic acid antigens. J Immunol 132: 872-876, 1984.

12. Stollar BD: Immunochemistry of DNA. Int Rev Immunol 5: 1-22, 1989.

13. Mohan C, Adams S, Stanik V and Datta SK: Nucleosome: A major immunogen for pathogenic autoantibody-inducing T cells of lupus. J Exp Med 177: 1367-1381, 1993.

14. Mamula MJ and Janeway CA Jr: Do B cells drive the diversification of immune responses? Immunol Today 14: 151-152, discussion 153-154, 1993 .

15. Routsias JG, Dotsika E, Touloupi E, Papamattheou M, Sakarellos C, Sakarellos-Daitsiotis M, Moutsopoulos HM and Tzioufas AG: Idiotype-anti-idiotype circuit in non-autoimmune mice after immunization with the epitope and complementary epitope 289-308aa of La/SSB: implications for the maintenance and perpetuation of the anti-La/SSB response. J Autoimmun 21: 17-26, 2003.

16. Poole BD, Scofield RH, Harley JB and James JA: Epstein-Barr virus and molecular mimicry in systemic lupus erythematosus. Autoimmunity 39: 63-70, 2006.

17. Pal R, Deshmukh US, Ohyama Y, Fang Q, Kannapell CC, Gaskin F and Fu SM: Evidence for multiple shared antigenic determinants within Ro60 and other lupus-related ribonucleoprotein autoantigens in human autoimmune responses. J Immunol 175: 7669-7677, 2005.

18. Terzoglou AG, Routsias JG, Sakarellos C, Sakarellos-Daitsiotis M, Moutsopoulos HM and Tzioufas AG: Linear epitopes of two different autoantigens-La/SSB and myelin basic protein--with a high degree of molecular similarity, cause different humoral immune responses. J Autoimmun 21: 47-57, 2003.

19. Chang SH, Huh MS, Kim HR, Kim IS, Kim S, Lee JS, Semsei I, Grölz D and Bachmann M: Cross-reactivity of antibodies immunoadsorbed to laminin with recombinant human La (SS-B) protein. J Autoimmun 11: 163-167, 1998.

20. Putterman $C$ and Diamond B: Immunization with a peptide surrogate for double-stranded DNA (dsDNA) induces autoantibody production and renal immunoglobulin deposition. J Exp Med 188: 29-38, 1998.

21. Scofield RH, Kurien BT, Ganick S, McClain MT, Pye Q, James JA, Schneider RI, Broyles RH, Bachmann $M$ and Hensley K: Modification of lupus-associated $60-\mathrm{kDa}$ Ro protein with the lipid oxidation product 4-hydroxy-2-nonenal increases antigenicity and facilitates epitope spreading. Free Radic Biol Med 38: 719-728, 2005.

22. Deshmukh US, Gaskin F, Lewis JE, Kannapell CC and Fu SM: Mechanisms of autoantibody diversification to SLE-related autoantigens. Ann N Y Acad Sci 987: 91-98, 2003. 